\section{Eine Damencrew für die zehnte Überwinterung in der Antarktis}

Im Dezember beginnt die zehnte, insgesamt 14 Monate dauernde Überwinterungsexpedition in der deutschen Georg von Neumayer-Station in der Antarktis. Zum ersten Mal gehören Damen zu einer solchen Expedition - und dann gleich die ganze Crew, denn "gemischte" Teams lehnt der Leiter des Alfred Wegener-Instituts (AWI) in Bremerhaven, Prof. Dr. Gotthilf Hempel, prinzipiell ab. Je zwei für Geophysik, Meteorologie und den technischen Betrieb sowie eine Funkerin, eine Köchin und als Leiterin eine Ärztin das war das übliche Fachschema auch bei den neun bisherigen Überwinterungen.

Für die Bundesrepublik haben sich dabei immer deutlicher Forschungsschwerpunkte auf den Gebieten Klima und Geophysik herausgestellt. Im Geophysikalischen Observatorium werden Feinstrukturen der Magnetosphäre und des südpolaren Elektrojets ermittelt; es ist ein wichtiger Punkt im weltweiten Beobachtungsnetz "Intermagnet". Globale Erdbebentätigkeit und Ermüdungserscheinungen des Eises werden im seismisch-gravimetrischen Observatorium gemessen. Das soll Auskunft über Struktur und Aufbau der Erdkruste und des oberen Erdmantels liefern sowie über den Zerfall von Kontinenten. Atmosphärische Untersuchungen (etwa auch des Ozonlochs) macht das meteorologische Observatorium. Atmosphärische Spurenstoffe in der Gegenwart und im Eis der Vergangenheit sind Thema des luftchemischen Observatoriums.

Neue Aufgaben der Damencrew sind der Aufbau einer Windkraft- und einer neuen Recyclinganlage. Die Leiterin der Expedition, die 34jährige Ärztin Dr. Monika Petra Puskeppeleit, bedauert, daß Polarmedizin bisher in Lehre und Forschung vernachlässigt werde. Ein Konzept „Humanbiologie und Medizin in Polargebieten" ist zwar seit Jahren vorgesehen, wird aber im AWI immer noch beraten.

\section{DFG fordert bessere NMR-Spektrometer und wissenschaftliche Infrastruktur}

Während die Kernspinresonanz(NMR)-Tomographie in der Medizin anatomische Bilder etwa von Läsionen des Gehirns anhand der Bewegung von Wasserstoff-Atomkernen im starken Magnetfeld (Nuclear Magnetic Resonance) wiedergibt und damit Veränderungen im Gewebe sichtbar macht, zeichnet die NMR-Spektroskopie mit Computerhilfe vor allem in der Chemie Strukturen von Flüssigkeiten, Lösungen und Festkörpern auf. Ihre Rolle für die medizinische Forschung am lebenden Organismus wird derzeit noch erforscht.
An mehreren Hochschulen in der Bundesrepublik fördert die Deutsche Forschungsgemeinschaft (DFG) solche Versuche. Schon diese ersten Ansätze haben deutlich gemacht, wie wichtig eine enge Zusammenarbeit zwischen Medizinern und Naturwissenschaftlern ist.

An den apparativen Möglichkeiten dafür aber fehlt es generell. Die "Mitteilung III" des DFG-Apparateausschusses (VCH-Verlagsgesellschaft Weinheim, ISBN 3-527-27374-3) zeigt die Richtung an, in die die Entwicklung von NMRSpektrometern gehen muß, und zwar zunächst bei den Lösungs- und Festkörper-Spektrometern für Chemie, Physik und Geowissenschaften sowie für Pharmazie, medizinische und biologische Chemie. Die Bedarfsanalyse für die In-vivoNMR-Spektroskopie als diagnostische Methode der Klinischen Medizin wurde vorläufig zurückgestellt. Die Anforderungen an die NMR-Spektroskopie steigen hier dramatisch, wenn nicht nur die Konstitution, sondern auch die Konformation und die Konfiguration eines Moleküls erforscht werden sollen.

Vor allem fehlt es an Spektrometern der höchsten Leistung von 500 und $600 \mathrm{MHz}$. Von den derzeit weltweit 260 Geräten in dieser Größenordnung sind die Hälfte in Nordameri$\mathrm{ka}, 26 \%$ in Europa und $22 \%$ in Japan installiert. Von den zehn europäischen $600-\mathrm{MHz}$-Geräten befinden sich zwei in der Industrie. In der Bundesrepublik verfügt die Gesellschaft für Biotechnologische Forschung in Braunschweig über eines der Geräte, das andere wird zur Zeit an der Technischen Universität München installiert.

Mit höchster Magnetfeldstärke - 11,7 Tesla und mehr sollen sowohl die Strukturen von Biopolymeren, komplexen Natur-, Synthese- und pharmazeutischen Stoffen sowie bestimmten anorganischen Verbindungen untersucht als auch Informationen über die innermolekulare Beweglichkeit gewonnen werden.

Zur Zeit sind in der Bundesrepublik 11 NMR-Spektrometer der erforderlichen Größenordnung installiert. Nach den DFG-Berechnungen müßten mindestens 22 weitere beschafft werden, davon vier $600-\mathrm{MHz}$-Apparate. Immerhin verdoppelt sich die Investitionssumme bei dem Sprung vom 500- auf den 600-MHz-Apparat beinahe, von 1,5 auf 2,7 Mio DM. Bisher hat die DFG 155 NMR-Spektrometer für mehr als 91 Mio DM beschafft. Solche teuren Geräte müssen allerdings sorgfältig regional verteilt sein. Auch dürfe man bei der sonstigen Ausstattung, insbesondere der Rechenkapazität, nicht sparen. Ob noch stärkere Magneten $750 \mathrm{MHz}$ - auf den Markt kommen werden, ist unklar; es dürfte noch mindestens fünf Jahre dauern, es sei denn, neuartige supraleitende Materialien machten einen schnelleren Durchbruch möglich.

Dringend sei ein interdisziplinäres Institut, das Fachkenntnisse der naturwissenschaftlichen Grundlagenforschung und der klinischen Anwendung verbindet, meinte der Naturwissenschaftler Prof. Dr. Dieter Leibfritz von der Universität Bremen vor der Wissenschafts-Pressekonferenz in Bonn. Er 